\title{
Acute circumflex artery total occlusion during ablation of septal premature ventricular contraction with radiofrequency energy
}

\author{
Krzysztof Myrda ${ }^{1}$, Krzysztof Wilczek ${ }^{1,2}$, Mariusz Gąsior ${ }^{1,2}$ \\ ${ }_{1}^{1} 3^{\text {rd }}$ Department of Cardiology, Silesian Center for Heart Diseases, Zabrze, Poland \\ ${ }^{2}$ Faculty of Medical Sciences in Zabrze, Medical University of Silesia in Katowice, Poland
}

Adv Interv Cardiol 2020; 16, 3 (61): 352-353

DOI: https://doi.org/10.5114/aic.2020.99276

The 28-year-old female patient was admitted to our clinic with symptomatic, frequent, drug-refractory ( $\beta$-blocker/propafenone) premature ventricular contraction (PVC) (Figure $1 \mathrm{~A}$ ). Both echocardiography and cardiac magnetic resonance showed normal values of cardiac chamber size and function, without valvular dysfunction. Using an electroanatomical 3D system (Carto 3 UniVu) and ablation catheter (Thermocool SmartTouch) (Biosense Webster, Diamond Bar, CA, USA), activation maps of both the right and left ventricles and the coronary sinus (CS), respectively, were created. Despite delivery of radiofrequency (RF) energy with 30-40 W to the interventricular septum from the left and right side the ablation was unsuccessful. Thus, based on the local signals, fluoroscopy view and 3D map, RF energy application was performed within the coronary sinus in the proximity of the posterior cardiac vein (MCV) (Figures $1 \mathrm{~A}-\mathrm{C}$ ). In the $60^{\text {th }} \mathrm{S}$ of the successful RF application with $20 \mathrm{~W}$, signs of ischemia were present in the 12-lead electrocardiogram. Urgent coronarography showed acute occlusion of the distal circumflex artery (LCX) (Figure 1 D). Successful wire crossing and recanalization were achieved with the coronary guide wire. Prolonged inflation with a $2.25 \times 12 \mathrm{~mm}$ semi-compliant balloon demonstrated a suboptimal result and therefore a $2.5 \times 15 \mathrm{~mm}$ sirolimus-eluting stent (Orsiro, Biotronic AG, Büllach, Switzerland) was implanted, with an optimal angiographic result (Figure $1 \mathrm{E}$ ). At discharge and in 6 months' follow-up, there was no evidence of recurrence of ventricular extra beats in 24-hour Holter monitoring. Based on the medical history, physical examination and the results of the additional tests, no signs of coronary artery disease were found.

Coronary artery occlusion is a rare complication of RF catheter ablation reported previously, with an incidence rate less than $0.2 \%$ [1]. Acute injury at this particular location is most commonly related to RF application during ablation of the accessory pathway in postero-septal localization or in proximity of the posterior interventricular vein (MCV) [2]. Most reports and a large group of studies describe occlusion of the right coronary artery (RCA) [2]. In the present case acute occlusion affected a branch of the left coronary artery - LCX - the dominant artery in this anatomical variant. Selection of ablation catheters and RF application settings in CS most often results from local experience. However, limiting power and duration of RF application, as well as choosing a different energy source (cryo), might decrease the risk of coronary artery injury.

In conclusion, we would like to emphasize that RF ablation in the ostial part of the coronary sinus/MCV is associated with a very small risk of occlusion/injury of RCA or CX depending on the coronary artery dominance.

\section{Conflict of interest}

The authors declare no conflict of interest. 

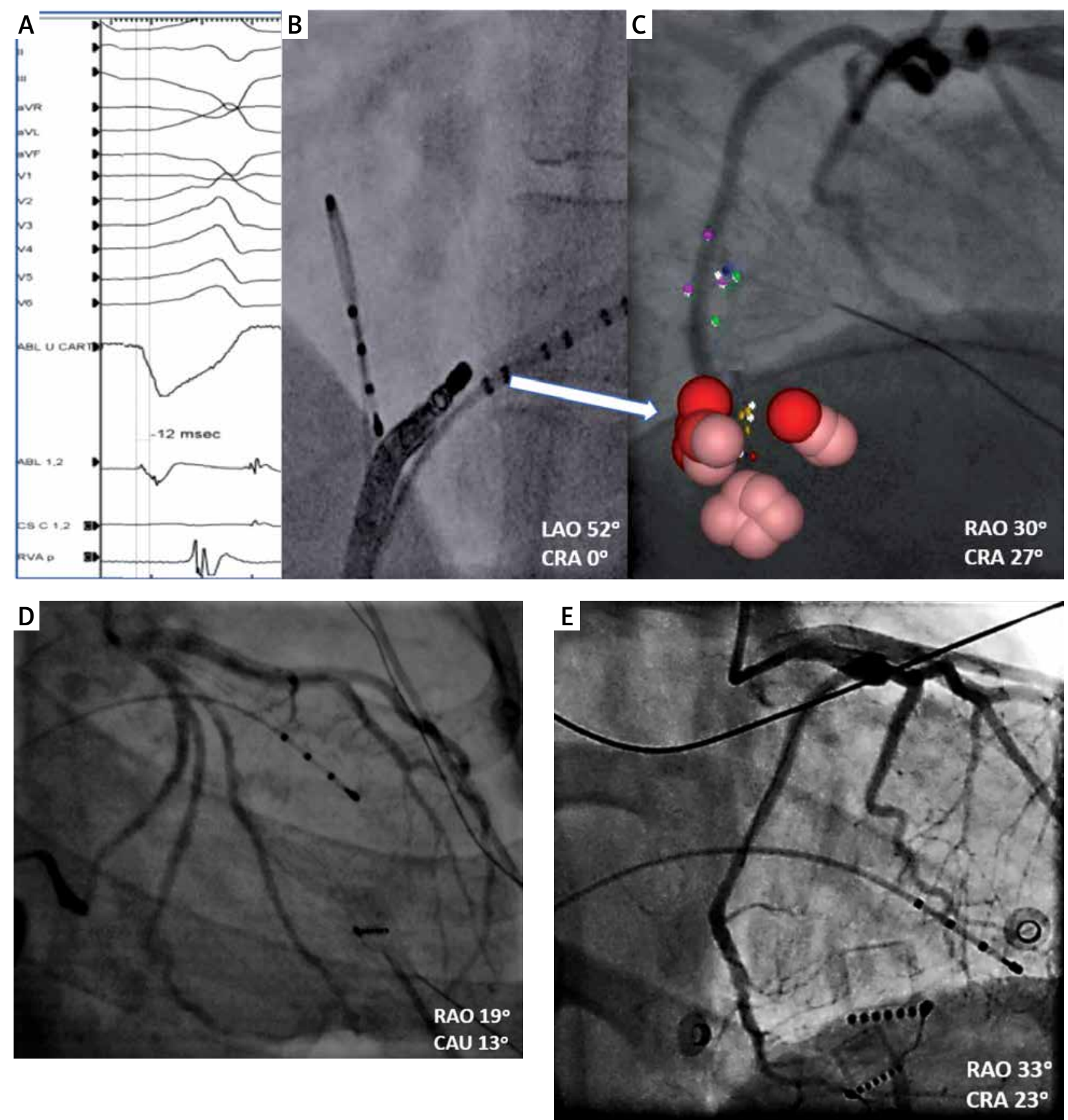

Figure 1. A - Recording of clinical arrhythmia, B, C - field of RF applications, D - occlusion of the distal LCX; decapolar catheter placed in CS, E - restored TIMI 3 flow in occluded artery

\section{References}

1. Roberts-Thomson KC, Steven D, Seiler J, et al. Coronary artery injury due to catheter ablation in adults: presentations and outcomes. Circulation 2009; 120: 1465-73.

2. Pothineni NV, Kancharla K, Katoor AJ, et al. Coronary artery injury related to catheter ablation of cardiac arrhythmias: a systematic review. J Cardiovasc Electrophysiol 2019; 30: 92-101. 